41.

\title{
liedjtsne/didjte.
}

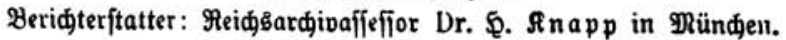

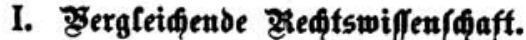

1. Rohler, Jof., Radträge zum Redt ber Serero und

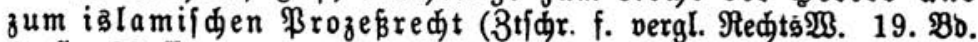
1. Sू., 29 ff.).

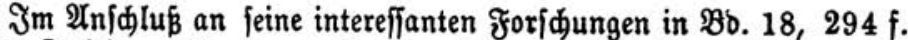
bringt Rogler wiffenșmerte 3 ujäkze, fo u. a, baß Blutrade aud bei Selbftmoro begrünbet ift, indem fich jene bann gegen ben Beranlaffer beşfelben ridtet, wie baß nod) bei Rebzeiten bes tötlid Berleşten ftattfindet, wobei biefer feinen Feino uno mutmaß̧liden Täter vor fid zu zitieren vermag.

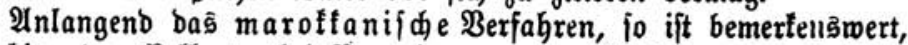
Daß̧ bier ber Beflagte bei Bermeigerung ber 'Einlaffung einfach in Faaft genommen wirb, baß̄ bie 3eugen auf ibre finnlide $\mathfrak{B a h r}$ nehmung nidbt befdräntt finb, fonbern aud gerabezu als urteiler fungieren fönnen, $\mathfrak{b}$. h. fich Gierüber erflären, was ihnen in ber Sadje Daş Ridtige zu fein fdeint. Der Eio ift nidt Tatjadjen= fondern Uniprudazeib. Frerner hat fich in Marofto ber Stand ber थिnwälte

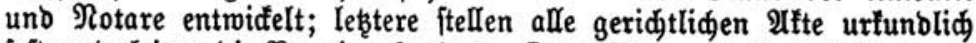
feít und leiten bie Bemeizanufnabme. Dem Släger ifft Selbijtbilfe burd Pfanonabme geftattet. Behörent bie Beteiligten veridieoenen Be= meinden an, fo greift momöglid aud) Blutrade plaß̧; andernfalla erlebigt baś ignen gemeinfame Dorfgeridgt ben Streit: man ruft bie

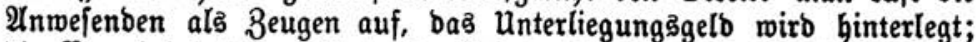

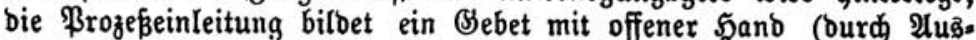
cinanderlegen ber Şändo nach oben und breimaliges überítreiden beą

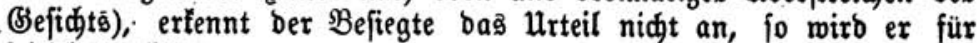
frieblos erflärt.

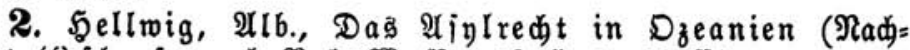

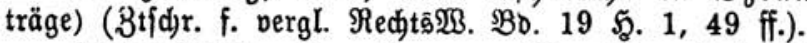

Seine Etubien bezmedten neue Şinmeife auf bas Beftehen beả Berbredjer=, Fremben=, mie, auf rcligiöler Brunblage fuß̧end, bę Tier $=$ und Bflanzenajy!s in Dzeanien. Die Darftellung bringt mand neue Ergebniffe, indem Berf. fid vielfad beftrebt, bie veridiebenen

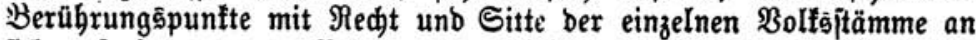

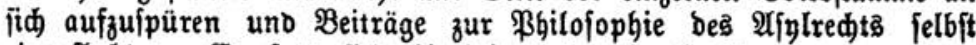
einzufledten. (Er feķt fid) hierbei aud mit Robler uno anbern Foridern außెeinander und nüß̨t bie Bselegenbeit, veridiebene frübere

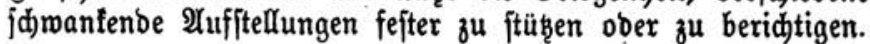


3. Radob, D., Befdidte von Japan (Rampredt, Beid. b. aupereurop. Staaten I) Bo. 1, 1, Die $\mathfrak{H}_{\text {rzeit, }} 426$ S., (Sotha, ßerthesె, 1906.

Die erfte wiffenfdaftlide Befdidte Sapans in beutider Eprade. Wäbrend bie Einleitung Lanb und $\mathfrak{B}$ olf, forcit fie für bie $\mathscr{A r b e i t}$

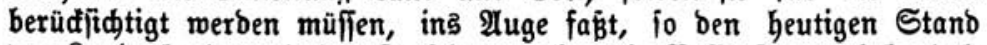
Der Ranbesłtunbe unb ber Frorfdung über bie Raffenfraue, behanbelt ber midtigfte Teil beả Buđhesె bas̄ halbhiftorifde 3citalter. ütber bie Rect) überliefert. Sie trägt, wie baş Staatsెmefen felbft, fairalen Sharafter. Tan tennt al̄ Sühne Reinigungäopfer und in fímeren f̧ällen Ber. Iuft von frreiheit, Leben und S̈ütern. IIIs Beweisfałtoren bienen

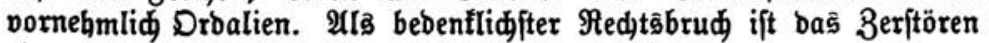

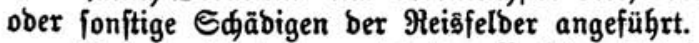

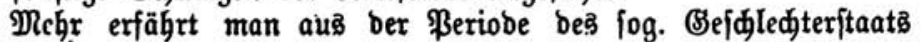
(Uji=ßerfaffung). $\mathfrak{A} u \dot{d}$ hier hängt inbea bie Beftrafung mehr ober minber von ber \&aune und $\mathfrak{B}$ illfür ber Şerrider $a \bar{b}$; bies bezeugt,

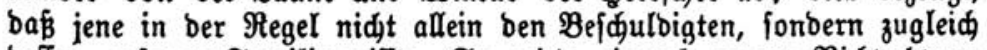
beffen gefamte framilie trifft. So wirb einmal wegen Nidtadtung

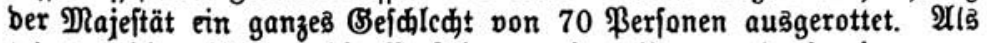
tobes̄mürobig gilt aud bie Berfübrung einer U1neme, b. h. einer zum

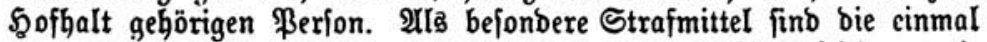

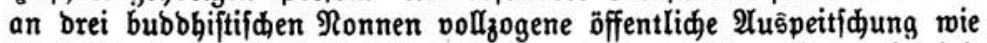

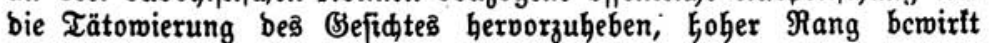
mcift Tiloerung ber Sühne. Tobeșftrafe fteht aukerbem nod auf Pord, গaub und Rerfübrung. Der Dieb mujte bas Cintwendete er= fezen ober fein Sos $_{\text {ar }}$ Berftlavung. Bielfad erfannte man aud auf Berbannung. Behufa Ermittelung von ßerbrchen bienten bie folter

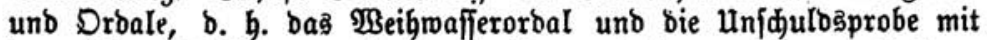
ber giftigen Sdilange, welde man, ohne verlełt zu werben, aus einem tiefen Topfe hervorziehen muß̧te. Bur Şinridtung von Tiffetätern war allein bie Reibgarbe beả Şerridersె befugt, in beren Tafde bann wohl auর basె Bermögen beä Beriđ̄teten ganz ober teilmeife glitt.

4. Eoulin, 21., Der geridtlide 3 weifampf im altfran=

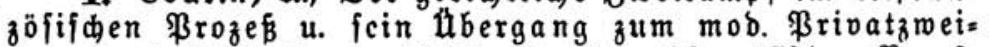
tampf. 1. Teil: Der geridttide $3 \mathrm{~m}$. im altfranzöfir Mit einem Borwort von Sofef Rohler. 169 S., Berlin, Butten= tag 1906.

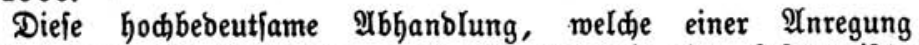
ร. Soblers ihre Entitegung verbanft, bezredt eine inftematifdec

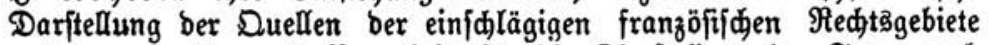
uno zwar miebcr al\$ Borarbeit für bie Rlaritellung ber Frage nad) bem Bufammenhang zmifden bem geridtliden und mobernen 3wei= fampf. W3ohl nod nie wurben bie Bejonberheiten bes alten und emiajungen Drbalä in fo anjdaulidjer $\mathfrak{B}$ eife vor $\mathfrak{X}$ ugen gefül)rt -

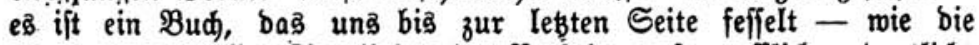
mitunter originellen Einzelheiten bes Berfabrens fo trefflid verbeutlidjt. 


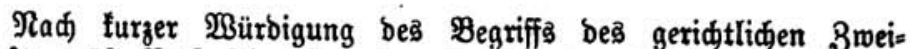

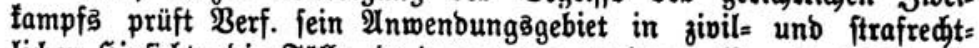
lider Şinfidt; bie Fällle, in benen man zu jenem Bemeisెmittel griff, finb äußerif zablreid. Şierauj gebentt er auşfübrlid ber perfönliden Borauşę̧ungen auf feiten ber beiben $\Re_{a ̈ m p f e r}$ in bezug auf ihre

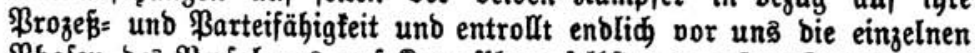
\$hafen besె \$erfabrens auf Rampftlage felbft vom erften Rampfes̄gruß

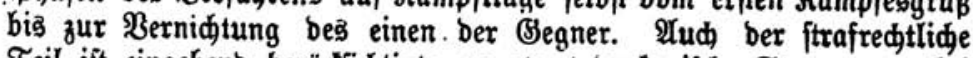
Teil ift eingehenb berüdfiditigt; er atmet bratonifकe Strenge, mobei (i⿱亠凶禸 Die graufame Sübne aud auf bie Rampfitellvertreter unb bie Bürgen eritreçt unb bie befiegte frau bem furdstbaren Freuertob ober bem Rebendigbegraben verfällt. Reiber ift ein Sidvertiefen in bies intereffante Thyema hier nid)t ermöglidst; nad ben bişher fo exfolg= reiden Stubien zu fdliefen, wirb es bem 2lutor fider in Päloe ge= lingen, bas erwünfidte, erftrebenswerte Biel zu erreiden.

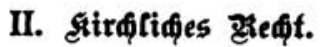

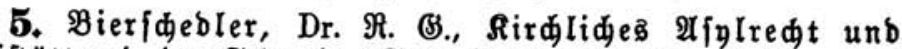

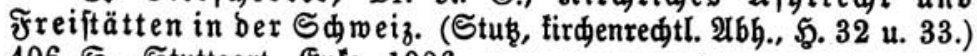
406 S.. Stuttgart, Enfe, 1906.

Diefe auf grünblidicn Borjtubien aufgebaute unterjudung ent=

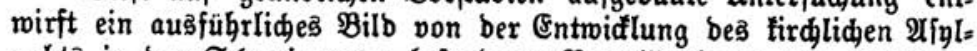

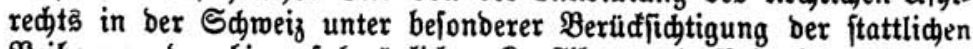
Reihe von ben hierauf bezügliden Ronfliften und Beftrebungen, jene

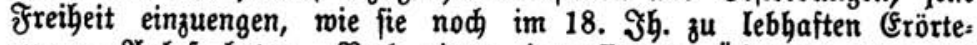

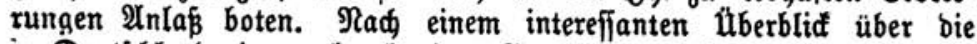
in Deutidland i. a. obmaltenden Brrundiäze, ftellt eả ber $\mathfrak{B} e r f$. für

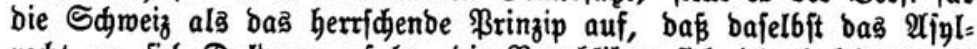
redt an fid Dulbung exfuhr, bic Republiten fid jebod feineșmegs

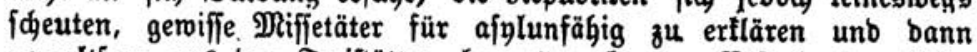

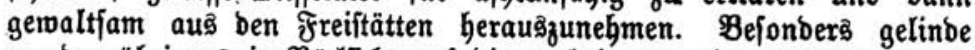

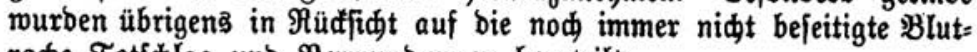
rache Totíliag uno Bermundungen beurteilt.

Arbgejehen von ben Sirchen felbft fteht vor allem ben friebböfen

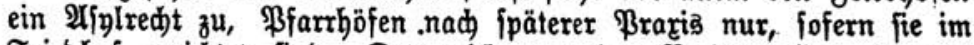

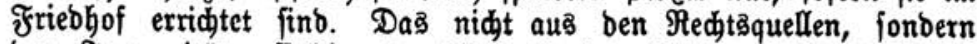

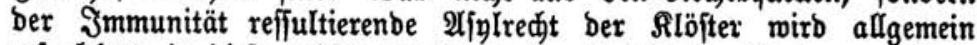
refpefticrt, ja biefe zählen gerabe zu ben beliebteften Freiungen. Înd in anfdaulidfter Weije führt unß ber Berf. bie hier maß̈gebenden

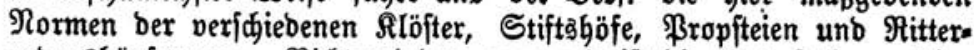

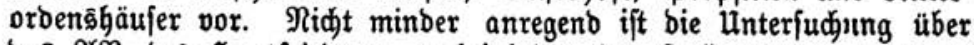

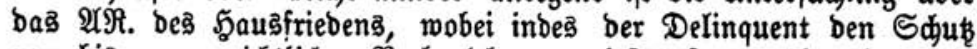

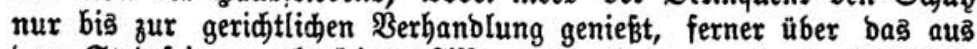

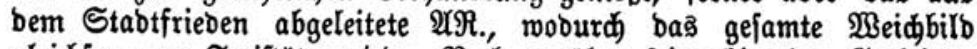

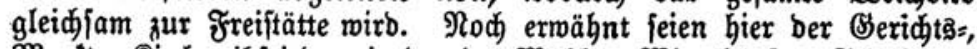

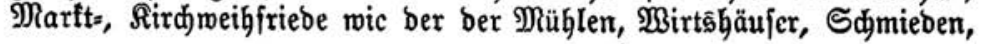


Babjtuben ujw. Eine bebeutfame Rolle fpielen fobann bie Dings und

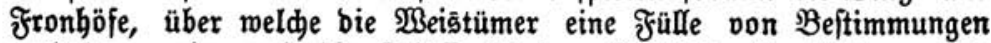
enthalten, wie aud bie Sdöffenbäuler, B̧eridtşs, Bunfträume uno fonftige freiböfe. Şervorzubeben ift aud bas $\mathfrak{A} \Re$ ber Şerridaft Sুal= benftein, bas erft im 17. Şh. fraft taijerl. Privilegş entjtand.

Trok bes an fid afnlfeindliden Eharafters ber Heformation be= ftehen in ber Sdwreia bie meltliden und geiftliden Freiftätten fort.

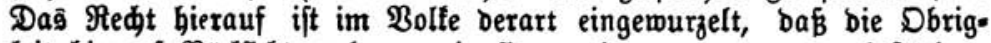
feit hierauf Rüdfidst nebmen, ja jie gerabezu von neuem beftätigen muß. Nur bas $2 \Re$. ber Sirden an fid verjळminbet, bebeutenden Siöftern vermag bie Reformation feinen Fintrag zu tun. Sn ben fatbolifiden (bebieten aber erbalten fid, ba bas Tribentinum baą $\mathfrak{A} \Re$.

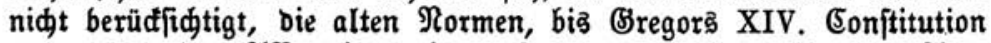

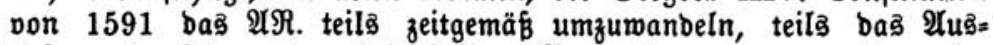
lieferungŝverfahren Der afylunfäbigen ßerbreder genau zu oronen bes

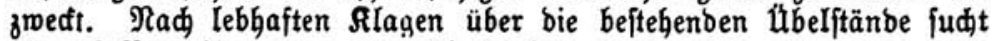
hierauf Beneoift XIII. 1725 bie bişherigen crimina excepta cettenfí zu exläutern unb außerbem neue Delifte für afylunmürbig zu extlären. Dod bie Ronflifte enben nidt, was weitere Berbandlungen ber evangelifden und fatholifđen Stände über (Finjd)ränfung ber fird).

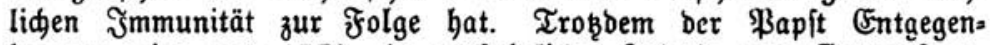
tommen zeigt uno 1770 ein auşführlideš ร̃nbult zum Entrourf ge= langt, verlaufen alle Beratungen ergebnișlos. Das $\mathfrak{X}$. über= lebt fid) endlid von felbjt, von bem Bseift ber 1798 beginnenden

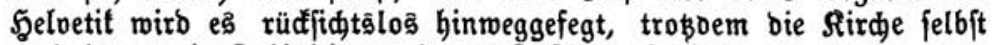
nod) heute mit Bäbigfeit an jenem Ĵnftitute feithält.

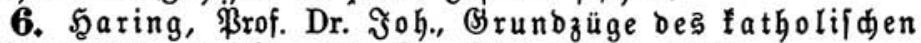
Rirdenredts. 1. Mbt. 310 S. Braz, Vtojer, 1906.

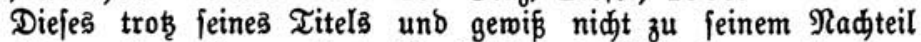

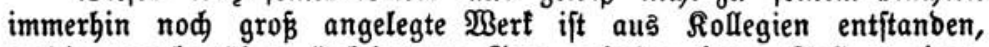
meldhe Der bemährte (Selehrte zu Braz gehalten hat. Soll eả einer= feițె zur Einfübrung beả angehenden Theologen in bie Rirdjenred)tsิ= miffenfdaft bienen, fo huldigt es anderfeits aud bem praftifden 3med,

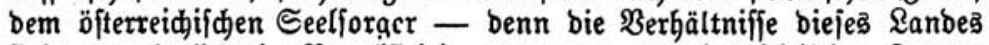
find vornebmlid in Berüdfidftigung gezogen - in fritifden Fragen förbernb unter bie 2 rrme zu greifen. Der vorliegenbe erfte Teil um= fajpt nad einer fehr łlaren Berbeutlidjung ber jurifitifden, theologifden und firdenpolitifien Borbegriffe einen furzgebrängten geididjtlidjen

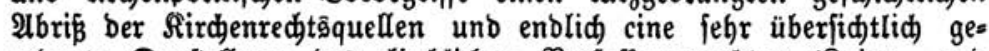

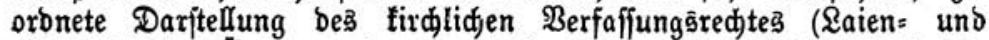

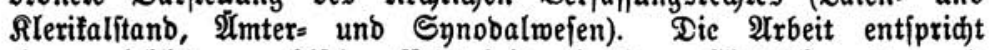
einem wirfliden praftilden Bedürinis̄; ein furzer Finbliđá genügt und bas, maవి in jebem fall als wiffensิwert erfdeint, tritt in reider

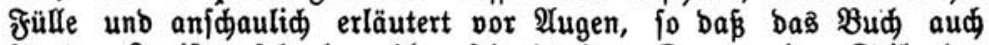
für ben Juriften fehr braudbar fein bürfte. Dem zmeiten Teil, ber bas fircliche Berroaltungäredft umfdhlieft, fann mit Spannung ent: gegengefehen werben. 


\section{Dentf́įes żewt.}

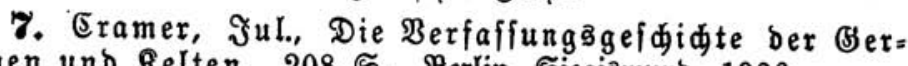
manen unb Relten. 208 S. Berlin, Siegismunb, 1906.

Diejer intereffante Beitrag zur vergleidjenden AItertumăunbe prüft, auf (Eäfar und Tacitus fukenb, zuerft bie Bejonderheiten ber beiben ßölfer, um bann bas ignen Bemeinjame feftzuftellen und aū̂ ber Blleidartigfeit ber ftaatlident Formen ben Nadweis ber nahen

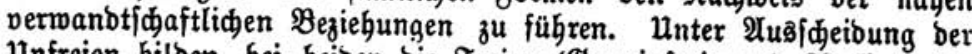

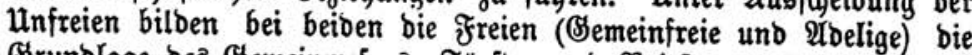

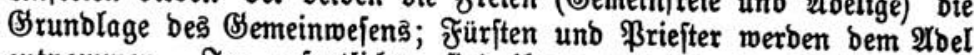
entnommen. Im mejentlidjen fino ihnen aud bie vier Stufen: Bses meinbe, Sুundertidaft, Taujerbidaft (Bau) und Bebntaufendidaft (Stamm) gemeinfam, ferner bie Baufürften, Füritenrat und Stammvers jammlung, Stammfüriten und Rönige, Şeereşformationen (Reil, \$halant, Mif fideben jebod bie Religionen und Rulte. $\mathfrak{B a s}_{\text {bie }}$ Radridten über

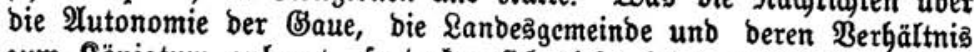
zum Rönigtum anlangt, fo beđen fid biefe bei beiben völlig. Şดre

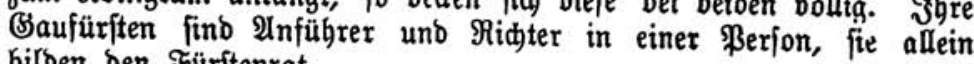
bilden ben Fürftenrat.

So zeigen fid bei Bcrmanen und Relten biefelben Bsrunbzüge ber Befellfdaftş= und Staaţorbnung, weld leştere fid auf bem Dezimalfyitem aufbaut; maßggebeno bleiben fie für jene bis zur

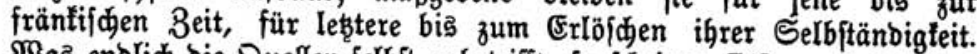
$\mathfrak{W a s}$ enolid bie Quellen felbft anbetrifft, fo fimeinen હäfar und Tacitua ziemlid gleidwertig; jeber bringt Sdjilberungen von Buftänben unb Beridjte über geididitlidje Ereigniffe, weldje teils grundlegeno, teils erläuterno erft in ihrer Bereinigung zu einem befriebigenben Befamt= billo führen.

8. Rofenthal, E্buarb, Bืefdidte bes Bseridtsmefens

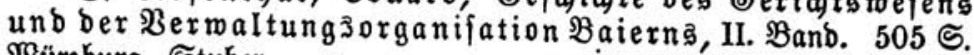
Würzburg, Stuber.

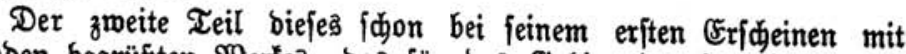

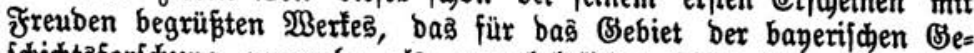

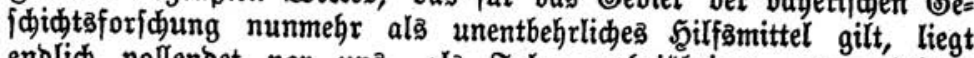
enolid vollenbet vor uns, als Folge mebriäbriger, unermüblidjer Stubien. Nur wer felbft mit ben hier einjolägigen Beftänoen ihrem wefentliden Jnḩalt nach vertraut ift, weiß zu ermeffen, wie verifiebenartig und fpröbe ber zu verarbeitenbe Stoff unb wie fómierig es ift, bie überall verftreuten maßgebenben $\Re_{0 r m e n}$ zu einem einheit. liden, übcrfidttliden ङanzen zu einen. Pit befonberer Sorgfalt if́t bie uns vor allem intereffiterende Beridts̄oerfaffung behanbelt. Sit

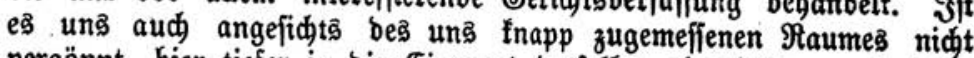
vergönnt, hier tiefer in bie Eigenart berfelben einzubringen, fo feien menigitens bie ફ̧auptzüge berfelben näher beleudjtet. 


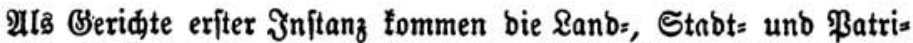

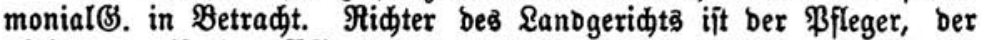

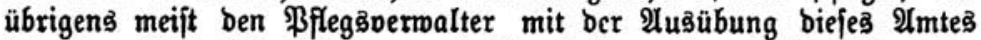
betraut. Rac Eibes̄leiftung un Bannleihe gilt now bie öffentlide

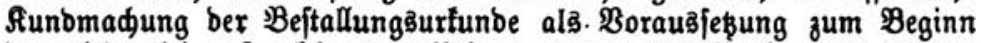
ber ridterliden fouttionen. Bei tobesెmürbigen Berbreden hat ber

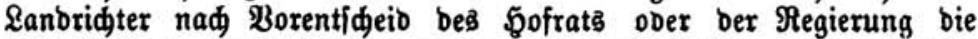
Unterjudung burdjufügren; bann gehen bie $\mathscr{A}$ ften an ben Bann=

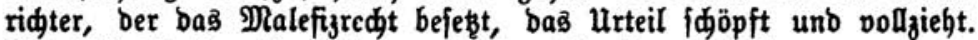
Bei Reibeôftrafen überläßt man in ber Regel ben Bollzug von vorn=

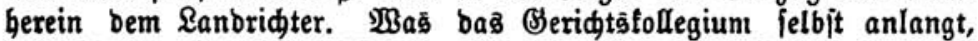
fo zäblt ber Sdreiber feit 1340 zu ben ftänbiaen Mitgliebern; bie

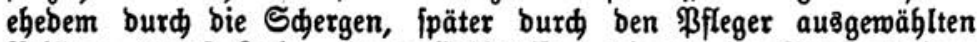
Beifiz̧er (zuerjt Jabrs, bann Erbjaofffen) bienten im Strafoerfahren

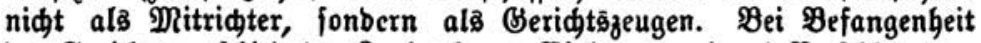

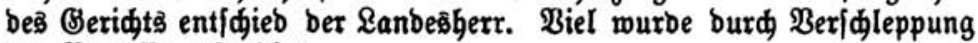
ber ßrozeffe gefündigt.

In ben Stabtgeridten fungierte ber Ridter früber als Drgan ber Stabt, wie bes \&anbesherrn, Enbe bes 16. 3h. wurbe inbes ben Fauptftäbten aud bie Striminal Sbtt. übertragen. Der Stabtridter hatte ben Eib vor bem Stabtrat wie ben Banneid vor bem Daundener Fुofrat zu leiften; fein Stellvertrcter in gröfieren St(S). war ber wo=

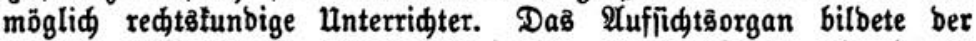
Stabtrat. PReift erfreuten fich bie Stäbte nur-ber Bivil= unb niebern Strafßsbtt; alle Bemohner und Brunoftüde innerbalb beạ : Burgfriebens', wie aud bie ङ̈äfte (Sdulbfacen) waren bem StङS. untermorfen. Da:

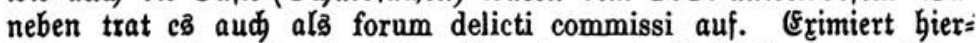
von waren bie lanbeŝherrliden Beamten uno Beiftliden. Nad, ber BrD. von 1616 burften bie Beifį̧er, wenn fie biōber nidłt nur alş

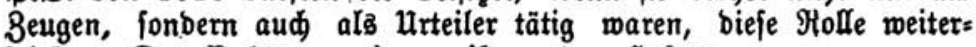

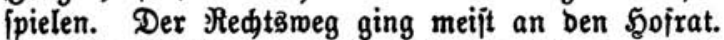

Die \$atrimonialङbft. exftređtte fid auf alle zur Sুofmarf ge=

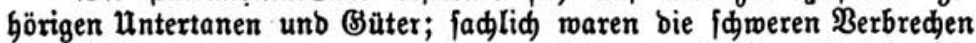
uno Bił̧tumbänbel aus̆genommen; nur felten waro einer 2 loelşfamilie

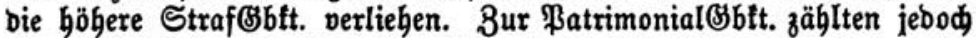

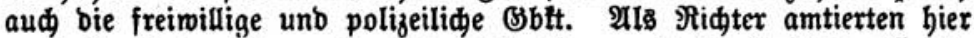

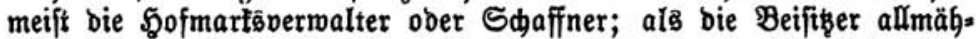
lid abfamen, übten fie bie volle Bemalt ber Einzelridjer. Dem fürits

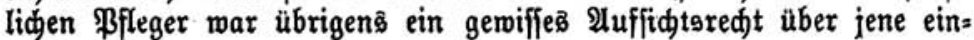
geräumt.

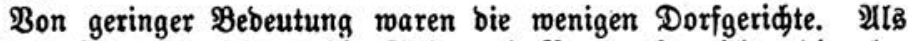

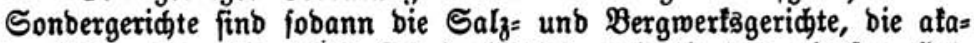

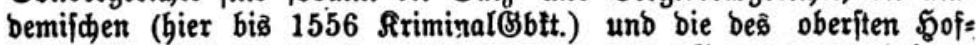

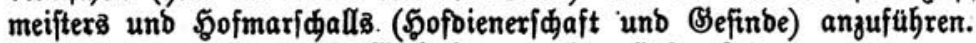
Unter Leţterem ftand als Crefutivorgan ber Fुofprofos.

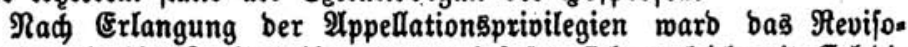

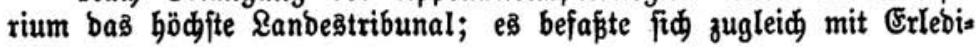


gung ber an ben faurften geridteten Supplifationen. IYnfanga fiel

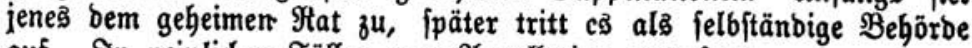
auf. In peinliçen f̧ällen war $\mathfrak{A} p$ pellation unterjagt.

Dem Fुofrat wie ben Regierungen war, was igre Tätigfeit auf bem Bsebiet ber Siriminaljuftiz anlangt, bie fकweren ober ßiştum=

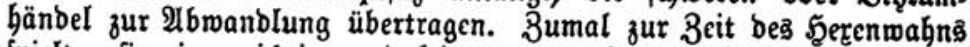

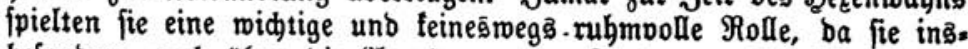
befonbere aud über bie 2 norbnung ber Tortur zu befinben hatten. $\mathfrak{A} u f$ feinen $\mathfrak{u}$ mritten hatte Der Rentmeifter alle Delifte aufzulpüren,

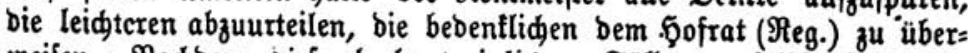
weifen. शadbem biefe hodnotpeinliden Fälle oortjelbjt tlargelegt

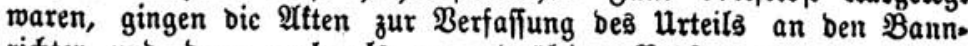
ridter uno bann nodmals zu endgültiger ßrüfung an bie höhere

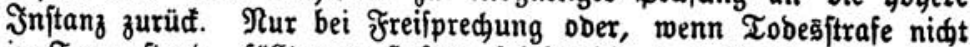

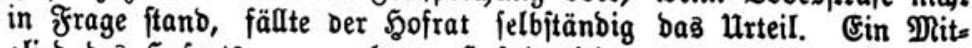
glieb bes Sूofrats war aud Der Sूofoberridter, bem Das \$olizei= unb ઉefängnis̄mefen ber Şauptiftabt unteritand. Daß von Fुofräten ge=

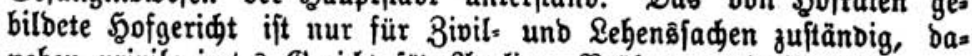
neben privilegiertę B̧eridjt für 2loelige, ßrälaten unb Beamte.

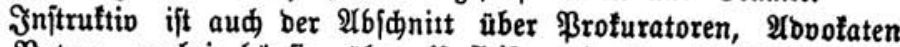

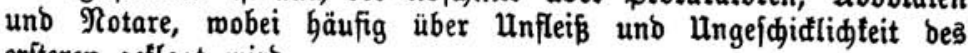
erfteren geflagt mirb.

Diesె zu furzem Einbliđ in baş reidbaltige ßert, basె jebem

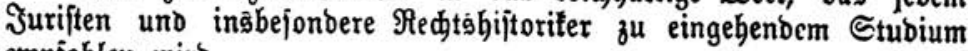
empfohlen mirb.

9. Liebegott, Martin Dr., Der Branbenburgifde Lanb.

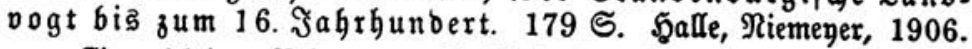

Ein widtiger Beitrag zur Bejdidte ber Daarfen. Währeno borts felbft bie erfte georonete Berwaltung in 21nlegnung an bie Burgen entitanlo, bildete fid in ben von ihnen entfernt liegenden $21 n$ fiebelungen Bogteicn. Seit Mitte besె 14 \h. werben nun ben Bogteien in ben fünf alten \$rovinzen Ranboogteien übergeorbnet. $\mathscr{A l s}$ ङrünbe Giers für gelten ber शangel an Rontrolle ben Bögten gegenüber wie bas

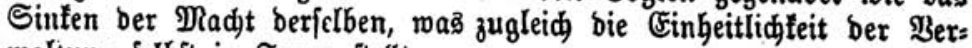
waltung felbjt in Frage ftellt.

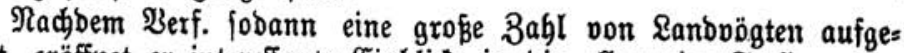
führt, eröffnet ex intereffiante Finbliđe in bie allgemeine Stellung ber

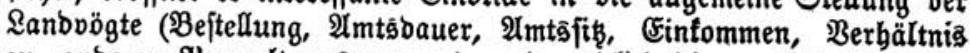
zu anderen Werwaltungsorganen) und enblid bie amtlide funttion berielben. Den Ranovögten obliegt als Bertretern Deả Ranbeşherm eine Dberauffiddt über bie Redtipredjung ihrer ßrovinz wie eine felbft=

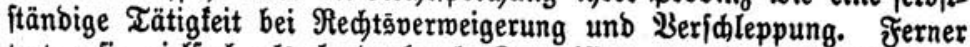
treten fie vielfad, als lanbeäherrl, Sommiffäre unb Sdjiebszridter auf; mitunter fübren fie aud ben Borfiţ im \$ूofo ober Ranbgeridgt. Sie

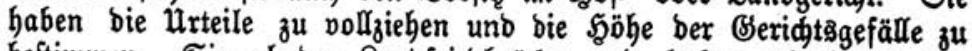
beftimmen. Sie ahnden Sanofrieobrüde uno haben überhaupt ben 
Lanbfrieben zu fdirmen, zumal auf ber Sুeerfiraß̧e, öffentliden \$räß̨en, ßüftungen und Deiden. Sie erbeben ftaatlide Einfünte, zumal ber geridttiden Bußen und fonftigen B̈efälle; fie nebmen Belehnungen vor und ziehen bie Rehenware an fid, fie unterftüz̧en bie Finanzbe= amten in ber $\mathfrak{A}$ uşübung ifgrer Pflidten. Enblid fommt ihnen bie hödffte Dilitärgewalt in ber \$rovinz zu; fie unternebmen Gier nidbt nur

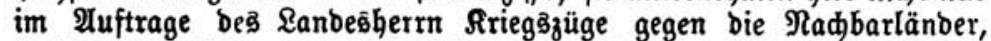
fonbern fie pflegen aud frieblide Berhandlungen mit biefen. Lanb= vögte und $\mathfrak{B o ̈ g t e ~ h a b e n ~ f i d ~ b i e r i n ~ g e g e n j e i t i g ~ B e i f t a n d ~ z u ~ L e i f t e n . ~ I n ~}$ ber Pittel=, Neumart und \$riegniz̧ tommen bie Ranobögte im 15. unb. 16. $\mathfrak{3}$ h. $a b$, in ber $\mathfrak{u} d e r=$ und $\mathfrak{A}$ ltmart ver[dminben fie erit im 18. Э̧ל. Die febr banfensెmerte froridung zcidnet fid burd B̧ründ:

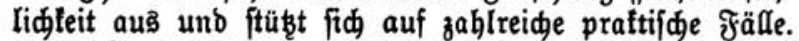

10. Stieber, Diloŝlav Dr, Dağ öfterreidif́de Land = redt und bie böhmif Rönig Dttofars in Defterreid (Dopid Dr. MIf., Foridungen zur

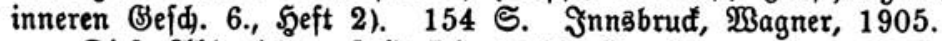

Diefe AXbhanblung ftellt fid als bie (Frweiterung einer $1901 \mathrm{er}=$ ¡dienenen böhmi

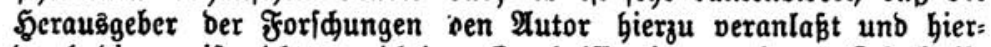
burd bie gemiß̧ nidjt unwidtigen Ergebniffe einem weiteren Referfreiie zugänglide gemadts hat. Stieber hat fid bie Âfugabe geftellt und bieje in befriebigenber Weife gelöft, nämlid bie Entjtehungą̧eit ber

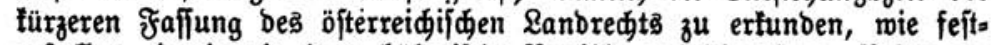
zuftellen, inmiemeit bą̧ böbmifide Borbilo auf bie öfterr. Reformen Dttofars eingemirft hat, und enolid eine Heberfidt über biefe ges ridtliden Reformen felbft zu geben. Das Bilb, weldes er von ber uriprüngliden Beridbtäberfaffung Defterreids entrirft, bifioniert in hohem Srabe mit ber hertömmliden $\mathfrak{A n f i d g}^{\text {; nad }}$ igm ift bie angeb=

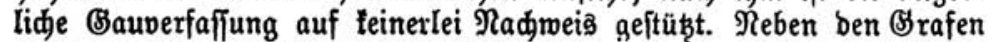
$g a b$ eả biefen unterftellte \&anbridter (judices provinciales), welde bie placita provincialia ober Eanbtaibinge hegten. J̧hre Sompetenz er= ftredte fid auf alle \$erfonen, melde nidyt vor ein höheres Frorum ober ben Stabtridter gehörten; Stifter uno Slöfter waren vielfach eximiert. Frühzeitig hob fid besె Martgrafen Bebeu=

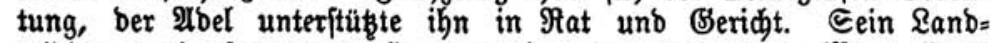

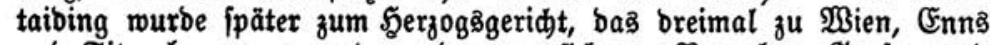
und Sitzenberg tagte und an bem an fich nur Bornehme, Srafen ullo

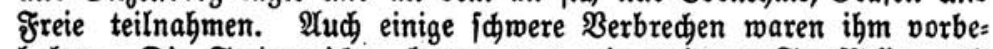
halten. Die Stabtgeridte hatten nur mit geringen Straffällen uno Rlagen um frabrbabe zu tun.

unter Dttofar bleibt ber Dbriftlanbridter beitehen, bod merben ihm zwölf Beifiz̧er beigefellt und für ihn eine felbftänoige Rompetenz gefdaffen. Sobann werben bie fog. obern Lanbridter in's Reben ge= rufen und ignen außer ber \&anofrage aud Bivilfadjen zugetwiefen.

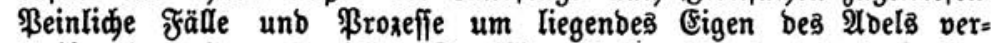

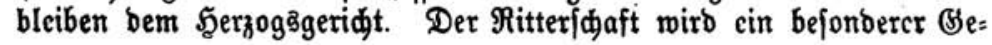




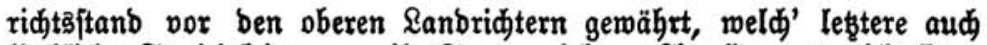

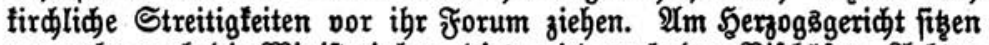

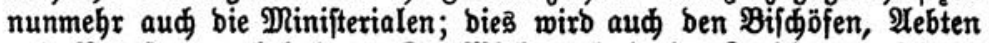
und \$röpften vorbehalten. Der $\mathfrak{B i r f u n g a ̈ t r e i s ~ b e r ~ \& a n b f r a g e ~ w i r b ~ e r = ~}$ meitert. Die Mactbefugnia bes Dbriftlanbridters fteigert fí mit ber Beit injofern, alä er bie peinliden Sadjen unb Slagen von Frahrbabe, fofern fie gegen $\Re$ tittermäfige geridtet finb, ben oberen Lanbridbtern entzieht. Daneben befteht ein bejonbereả Maridallägeridt. Pebeut= fam und in vieler Şinfint bem böhmifden ßorbild hulbigend äujert fíd enblid Dttofarä Ssefęzgebung auf bem widtigen SEebiete beả Stabtredtâ.

11. Boltelini, F̧ans v., Die Entftehung ber Ranbge.

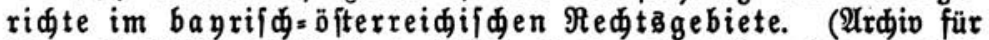

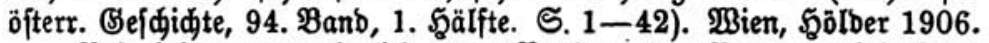

Brf. bringt, vornehmlid unter ßrüfung ber Bergangenbeit Süb=

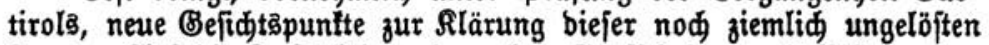
Frage. 2luf bie Berftüđfelung ber alten (Braffidaften und Bilbung ber Ranogeridite bat in erfter Rinie bie Bunahme ber Befieblung uno $\mathfrak{B e}_{e}=$

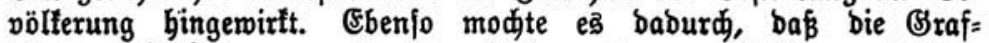

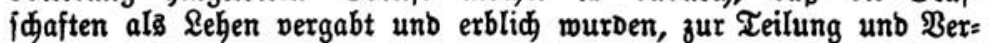
einigung biefer Bebiete fommen. Tiefgreifend zeigte fid aud zumeilen bie fönigliđe ßerleigung einer J̃mmunität, wie inābejonbere bie Erem=

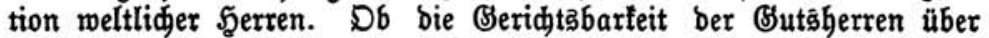

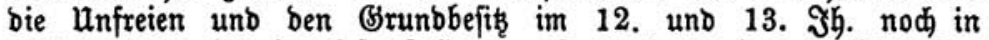
Beltung wax, ift nidt fejtzujtellen; an Stelle ber privatreditliden Ju= risbiftion trat vielfad ber Ermerb ber offentliden Beridgabarfeit innerbalb eines geidloffenen Bezirtä.

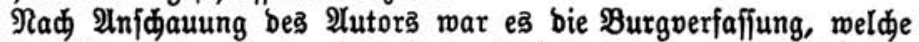
zur थuşbilbung ber \&anogeridte bas midhtigfte Ferment bilbete; bie Butgen traten in innigfte Berbinoung mit ben Raniggeridten. Der Burgvogt übte ben Burgbann über bie Bemohner beả Burgfriebens,

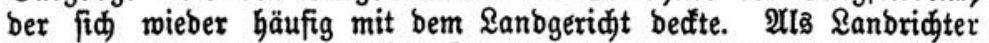
bejaß̧ ber Burghauptmann bie Rompetenz, welde ihm bem Burgbann gemäß zuftand; auß bem Burgfrieben entwidélten fid lanbesfürfitlide und patrimonielle Beridte. ßirffam erriejen fid alfo verjdiebene

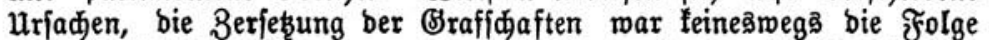

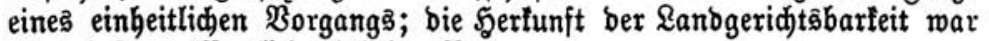
hierbei ftets öffentlidjredtlidjer Natur.

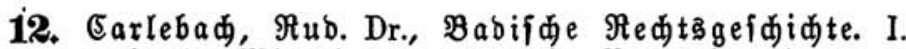
Das ausgehende Mittelalter und Die Rezeption bea rö=

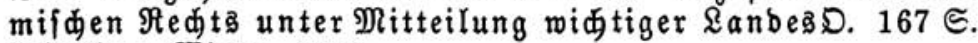
Şeibelberg, Winter, 1906.

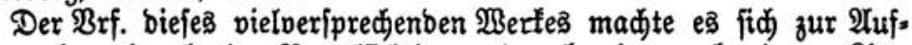
gabe, neben eingebender Berüđfichtigung ber bereitş vorbanbenen Sites ratur bie bisher nod im Rarläruber Esenerallandeảardio verborgenen einfdlägigen Dohumente zu Tage zu förbern und auf Bruno ber= 
felben bie Redjtägejdidjte feiner Fुeimat felbjt aufzubauen. Da ein

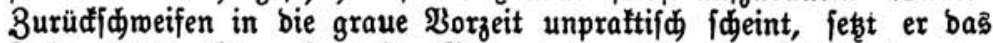
Jabr 1400 als entferntefte Srenze; im übrigen teilt er in vier Epoden ein: bas auşgehenbe Mittelalter und bie Rezeption, ber

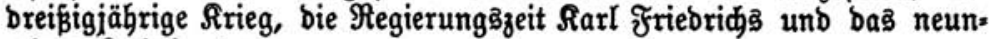
zebnte Sahrhundert.

Inlangend basิ uns vornehmlid intereffierende Strafredt wie bas Beridtşmejen, fo ermeifen fid in erfterer Şinfidt bie frübeften

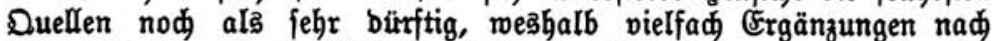
Dajgabe ber allgem. Rechtsggeididte benötigt find. Selbft über bie Inngeridte wie biejenigen, bie bierüber zu Beridt faßjen, fdpmeigen fid jene faft völlig aus. Mebr erfabren wir über bie geringerent Delifte: Die Frevel ftellen fid als tätlide Berlek̨ungen bar, bod ohne

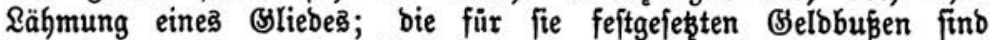

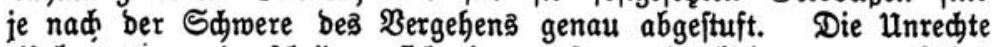
(Uebertretungen) (d)eiben fid in großße und fleine, man fühnt fie mit 15 und 5 Sdillingen. Ebenio merben bie Finungen (Ulebertretungen ber Dorfgefełze) mit Buß̉en, welde ber Bemeinbe zu= fließ̧en, geabndet. Şinfidtlid jener brei (Sruppen beftebt eine Rüge= pflidit aller, melde feine Renntnis erbielten. Friebbrudbsbelifte ges wärtigen bie bei ber Friebbietung feftgefeł̧te Strafe; Bumiberhand= lungen gegen lanbeahgerrl. Bebote beftraft man an Reib unb But naw fürftl. Cerrmeffen, wobei \$forzheim uno Baben für ihre Bewohner wi

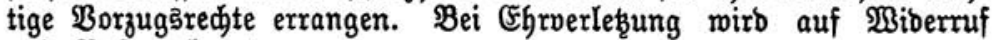
und Bufe erfannt.

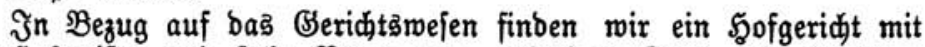

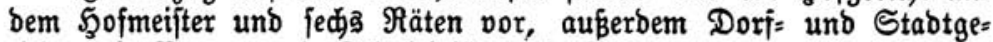

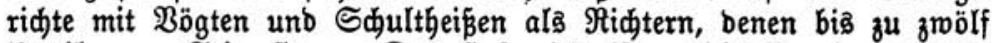

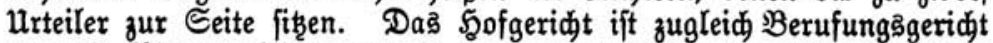
für bie Niebergeridte; nur in ben Saujenberger Seerridaften bejteht

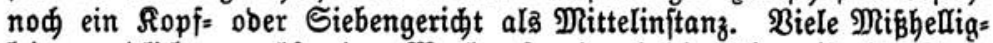
feiten enolid) ermudifen bem Marfgrafen burd bie Hebergriffe ber Feme

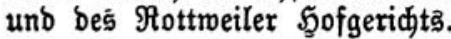

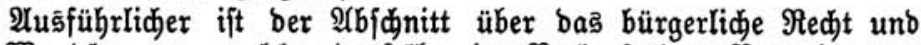
bie Bandlungen, weldhe başfelbe im Berlauf ber Rezeption er= fabren hat.

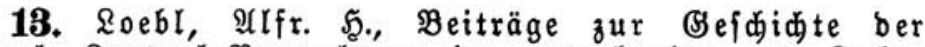
faiferl. Bentral= Bermaltung im ausgehenben 16. Jahr=

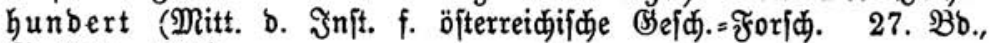
厄. 629-677.).

Diefe inftruftive Unterjudung entmirft ein ziemlid troftlofes \$illo von ber Bejdaffenheit ber beutiden Bentralbehörben, benen aus jeg= liđem Mangel an Berftänonis für bie iłnen obliegenden miđtigen Uufgaben ein weiter $\mathfrak{B l i t}$ wie ein großzzügiges Şanbeln völig ab=

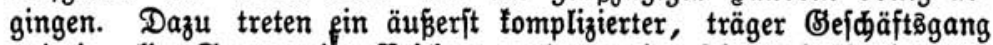

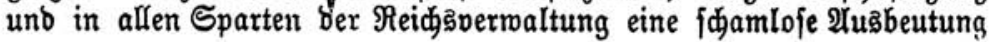




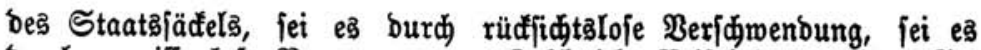

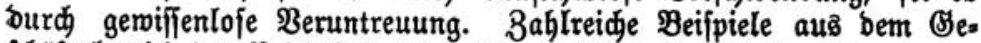

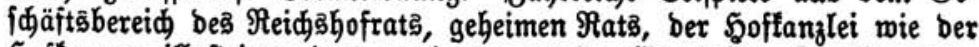
Fsoffammer ifluftrieren jene menig anmutenben Ergebniffe. Der Beamten. törper war bierbei ein meitverzmeigter, vielföpfiger und veriळieben= artiger; neben bem Stand ber Berufäbeamten bildete fid ein Berufb̄= biplomatenftand heraus, ber allerbingä, ba er ftänbifden Einflüffen burdaus fernftand, eine neue fürftlide Stütse barbot. Bei Charafteri= fierung biefer $\mathfrak{B}$ eamtenfकaft trägt nun $\mathfrak{B r f}$. bie büfterften frarben auf.

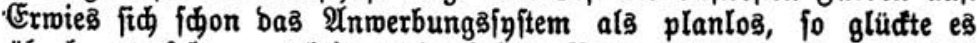

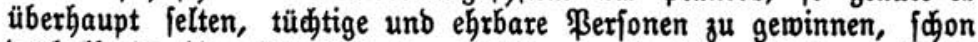

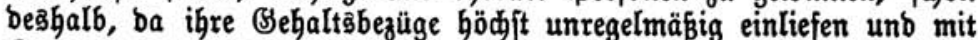
Bratififationen, Berpfänbungen von Staatßeintünften u. bgl. nidłtz gebient war. Rein \$unber, wenn angefidts einer faumfeligen Ron=

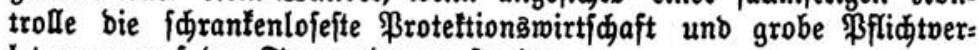
lę̧นngen auf ber Tagesెorbnung ftanben.

14. Rnapp, Dr. Şermann, Daş \&odgefängnis̄, Tortur

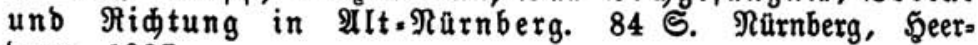
begen, 1907.

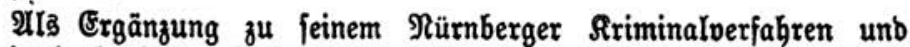
Sriminalredt bringt $\mathfrak{B r f}$. auf Brunb genauer urfundliळer froriळung,

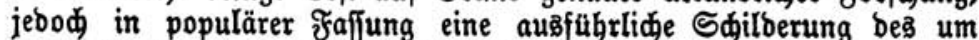
1340 in ben unterirbif \&odgefängniffes wie ber veridiebenen Sđjiffale ber in ih̆m einge= ferferten $\mathfrak{W} i$ iffetäter von ber peinliden $\mathfrak{U}$ nterfudjung an biaె zur \&ebenşs

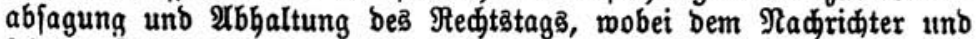

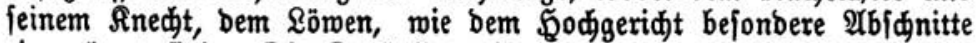

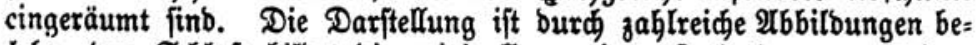
lebt, ben Sđluß bilbet bie originelle gereimte Rodjorbnung aus bem

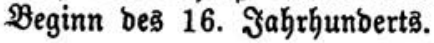

